\title{
Kernos
}

Revue internationale et pluridisciplinaire de religion grecque antique

$29 \mid 2016$

Varia

\section{Étudier les mythes en contexte francophone. À propos de quatre ouvrages récents}

\section{Philippe Matthey}

\section{Q OpenEdition \\ Journals}

\section{Édition électronique}

URL : https://journals.openedition.org/kernos/2405

DOI : 10.4000/kernos.2405

ISSN : 2034-7871

\section{Éditeur}

Centre international d'étude de la religion grecque antique

\section{Édition imprimée}

Date de publication : 1 octobre 2016

Pagination : 391-403

ISSN : 0776-3824

\section{Référence électronique}

Philippe Matthey, «Étudier les mythes en contexte francophone. À propos de quatre ouvrages

récents », Kernos [En ligne], 29 | 2016, mis en ligne le 01 octobre 2016, consulté le 15 avril 2022. URL: http://journals.openedition.org/kernos/2405 ; DOI : https://doi.org/10.4000/kernos.2405

Ce document a été généré automatiquement le 15 avril 2022.

Kernos 


\title{
Étudier les mythes en contexte francophone. À propos de quatre ouvrages récents
}

\author{
Philippe Matthey
}

\section{RÉFÉRENCE}

Claude CALAME, Qu'est-ce que la mythologie grecque ?, Paris, Gallimard, 2015. 1

vol. $11 \times 18 \mathrm{~cm}, 732$ p. (Collection Folio Essai). ISBN : 978-2-07-044578-3

John SCHEID, Jesper SVENBRO, La tortue et la lyre. Dans l'atelier du mythe antique, Paris, CNRS

Éditions, 2014. 1 vol. $14 \times 22$ cm, 229 p. ISBN : 978-2-271-07883-4

Pauline SCHMITT PANTEL, Une histoire personnelle des mythes grecs, Paris, Presses

Universitaires de France, 2016. 1 vol. 12,4 × 18,9 cm, 210 p. ISBN : 978-2-13-063481-2

Ute HeIDMANN, Maria VAmVoURI RUfFY, Nadège CoutAz (dir.), Mythes (re)configurés. Création, dialogues, analyses, Lausanne, Université de Lausanne, 2013. 1 vol. $16,5 \times 23 \mathrm{~cm}, \mathrm{x}+270 \mathrm{p}$.

(Collection du CLE). ISBN : 978-2-8399-1292-1

1 Qu'est-ce qu'un mythe? Quel sens lui donner? Quels sont les processus qui mènent à sa création, à sa diffusion, à ses transformations? Ces questions ne cessent d'agiter les esprits et d'intéresser le monde académique autant que le grand public, et les réponses que l'on y a apportées ne font pas l'unanimité. Parmi les innombrables ouvrages vendus dans les librairies du monde entier sous la rubrique "Mythologie» et inscrits dans plusieurs bibliographies des cours d'introduction à l'étude des mythes, ceux qui rencontrent le plus de succès auprès du grand public et des étudiants débutants sont toujours des traités et compilations mythographiques présentant les divers mythes grecs (ou égyptiens, scandinaves, etc.) dans leur version "pure » ou "idéale», en proposant des interprétations relevant le plus souvent de l'archéologie du XIX siècle, de la psychanalyse freudienne ou de théories inspirées par les archétypes jungiens. Une brève enquête à propos des meilleures ventes sur le sujet dans des librairies en France 
et en Suisse, par exemple, révèle un trio gagnant composé du manuel mythographique du poète anglais Robert Graves ${ }^{1}$, des travaux d'Edith Hamilton ${ }^{2}$ ou encore des théories de Joseph Campbell à propos du "monomythe $»^{3}$. Ces modèles d'interprétation sont complètement dépassés, voire n'ont même jamais été reconnus comme valables, mais il s'avère que les ouvrages de vulgarisation sérieuse venant du monde académique ont de la difficulté à rivaliser avec leur puissance d'explication.

2 La principale pierre d'achoppement dans ce domaine semble être la définition même de ce que serait un mythe et de ce qui le différencierait d'un autre type de récit, de forme de pensée ou d'objet narratif. Aujourd'hui, dans le grand public mais aussi dans de nombreux cercles académiques, la conception que l'on a du "mythe " est le plus souvent inspirée de la typologie proposée dans les travaux d'Arnold van Gennep et de Vladimir Propp, considérant le "mythe» comme une forme de récit traditionnel à portée sociale, distinct du « conte » et de la « légende »" Elle doit également beaucoup à l'influence de Roland Barthes et de Claude Lévi-Strauss, qui voient le mythe comme une certaine forme de pensée, une réalité transcendante qu'il serait possible d'étudier audelà de ses incarnations ponctuelles dans des récits ou des images ${ }^{5}$. Or, dans le domaine des études classiques et de l'histoire des religions, il est largement admis depuis les années 1980 que le concept de "mythe » est en réalité un leurre académique qui doit être remis en question, une invention moderne qui ne fait référence à aucune catégorie indigène, notamment dans le monde grec antique ${ }^{6}$.

3 Les quatre ouvrages recensés ici participent de ce consensus sur l' «illusion du mythe ", et leur publication au cours des dernières années témoigne de la volonté d'apporter un éclairage à la fois sérieux et compréhensible sur les questions relatives à la production et aux fonctions des récits regroupés sous l'appellation de «mythes ", ici plus particulièrement dans le domaine de l'Antiquité gréco-romaine. Deux d'entre eux rassemblent en réalité d'anciennes recherches et enseignements sur les mythes de l'Antiquité classique menées peu avant la fin $\mathrm{du} \mathrm{xx}^{\mathrm{e}}$ siècle par John Scheid et Jesper Svenbro (La tortue et la lyre. Dans l'atelier du mythe antique, 2014) et par Claude Calame (Qu'est-ce que la mythologie grecque?, 2015). Le troisième est un ouvrage collectif dirigé par Ute Heidmann et regroupant les discussions et réflexions menées sur le même thème par un groupe de chercheurs et d'artistes suisses entre 2000 et 2010 (Mythes [re]configurés. Création, Dialogues, Analyses, 2013). Le quatrième ouvrage, présenté par Pauline Schmitt Pantel (Une histoire personnelle des mythes grecs, 2015), propose quant à lui une brève vue d'ensemble de la mythologie grecque caractérisé par l'ambition d'être accessible au grand public sans pour autant renoncer à une certaine exigence académique. C'est sur ce dernier que l'on ouvrira le présent compte rendu, pour ensuite traiter les autres publications en remontant le fil du temps.

\section{Une histoire personnelle des mythes grecs}

4 L'ouvrage de Pauline Schmitt Pantel propose une approche de plusieurs mythes grecs présentés par l'historienne et helléniste française dans une optique de vulgarisation. L'A. évite cependant de céder à la tentation de livrer un ouvrage de mythographie érudite dans lesquels quelques résumés d'épisodes mythiques se trouveraient coupés de leur support textuel, de leur contexte d'énonciation et de leur pragmatique propre. Ainsi qu'elle le précise (p.11), son histoire des mythes grecs suit une « double ligne directrice : ne jamais perdre de vue le contexte dans lequel le mythe a été écouté, et 
tenter de comprendre les liens ténus mais réels entre ces récits et la construction du monde grec ». Dans le dernier chapitre (p. 179), l'auteure rappelle que son approche des mythes était initialement tributaire des travaux de Marcel Detienne et Jean-Pierre Vernant, mais qu'elle a toujours eu tendance à privilégier leur contexte historicoculturel à la suite de Pierre Vidal-Naquet et Nicole Loraux. Elle a également été convaincue par Claude Calame de la nature illusoire de la catégorie même de "mythe " et de l'importance d'étudier la mise en discours des récits de l'Antiquité pour mieux les comprendre.

De fait, l'ouvrage rend hommage aux études de Marcel Detienne et de Claude Calame dès son ouverture sur le rappel que "les Grecs n'ont pas disposé d'une notion correspondante à notre concept moderne de mythe»(p.6). Pour autant, l'auteure estime qu'utiliser le terme de «mythe » reste un moindre mal quand on tente de réunir sous un seul mot la grande disparité des formes de discours produits dans l'Antiquité grecque : elle propose donc une définition opératoire de la notion de mythe comme recouvrant: 1) des récits obéissant aux lois de la narration (inscrits dans une temporalité et un espace spécifiques); 2) mais aussi des histoires que l'on raconte devant un public (d'où l'importance de la performance et du contexte d'énonciation); 3) et enfin des récits se présentant sous la forme d'un texte, même s'il s'agit d'un texte transmis pendant des générations sous forme orale.

6 Après cette brève introduction sur les problèmes méthodologiques posés par l'étude des «mythes », le reste des 210 pages du livre est réparti entre huit chapitres abordant les thèmes de "La création du monde et la naissance des dieux", "Les débuts de l'humanité ", "Histoires de divinités », "Les mythes au féminin», "Mythes et Histoire », «Les mythes dans la cité », « Mythes et société » et enfin "Les mythes, de la Grèce à nos jours ». Dans les quatre premiers chapitres, les différents récits mythiques traités sont résumés de manière descriptive et à travers des extraits tirés principalement des poèmes homériques et hésiodiques, de la poésie lyrique, des tragédies classiques, mais aussi des recueils mythographiques, poétiques et des traités d'histoire de l'époque hellénistique et romaine. Fidèle à son projet initial, l'auteure présente brièvement, chaque fois que cela s'avère possible, le texte porteur de l'épisode mythique étudié et s'efforce de mentionner, même si ce n'est qu'en quelques mots, le contexte de production et d'énonciation de chaque source, sa fonction performative ou encore les intentions politiques du poète. Quelques intermèdes analytiques parsèment enfin le texte afin d'évoquer, toujours en appelant à la plus grande prudence, quelque clés de lecture communément avancées pour expliquer par exemple les rapports entre mythes et rites (l'Hymne homérique à Déméter comme mythe étiologique des Mystères d'Éleusis, p. 55-56), ou entre mythes et société (le mythe des races hésiodique comme parallèle aux prétendues fonctions sociales indo-européennes, p. 37). Dans les quatre derniers chapitres, l'auteure se concentre plutôt sur une esquisse du fonctionnement de la cité grecque (principalement athénienne) et sur ce que certains mythes peuvent nous en apprendre: rapports de genre, banquets, fêtes et tragédies, sacrifices et commensalité, éducation citoyenne, deuil et mort, etc. L'ultime chapitre est l'occasion de résumer la manière dont les Grecs et nous-mêmes avons lu et interprété ces récits, de l'Antiquité jusqu'à l'époque contemporaine, en prenant pour exemple les « mythes » de l'Atlantide et d'ÆEdipe.

7 L'ouvrage ne comporte aucune note de bas de page, sans doute pour ne pas rebuter le lectorat non-académique, et les différents textes sont illustrés par plusieurs extraits 
présentés dans leur traduction française empruntée à la collection des «Belles Lettres». Le volume comprend une liste chronologique récapitulant les auteurs antiques cités, un bref index des noms de personnes et une bibliographie succincte de quatre pages, centrée presque exclusivement sur des recherches issues du monde francophone mais incluant les meilleures publications susceptibles d'aider le néophyte dans sa découverte de la mythologie grecque et des théories interprétatives possibles. Cette histoire personnelle des mythes grecs constitue donc une introduction salutaire aux récits de l'Antiquité grecque, et sait rester accessible au grand public tout en le sensibilisant au fait que "les mythes ne sont pas saisissables sous une forme "pure", "canonique", "éternelle", comme les dictionnaires de mythologie ou les anthologies les présentent trop volontiers » (p. 7-8) : les mythes ont bel et bien une histoire propre.

\section{Qu'est-ce que la mythologie grecque?}

8 Publié dans un format poche, l'essai de Claude Calame donne au premier abord l'impression, comme le livre de Pauline Schmitt Pantel, d'être adressé au grand public. C'est toutefois loin d'être le cas. Ainsi que l'A. le précise dans son avant-propos, l'ouvrage aurait en réalité dû s'appeler (au pluriel) Poétiques des mythes dans la Grèce antique, en référence à la Poétique des mythes dans la Grèce antique (Paris, Hachette, 2000) dont il est une réédition augmentée (732 pages au lieu de 286) et en partie réécrite. Dans cette première Poétique des mythes dans la Grèce antique, l'A. disait répondre à « une commande motivée par le souci pédagogique et la nécessité d'informer un public d'étudiants cherchant à s'initier à la culture hellène dans la lecture des textes ${ }^{7}$. Mais malgré ces louables intentions, cette nouvelle version semblera sans doute encore trop complexe aux yeux de la majorité des lecteurs, du moins ceux qui ne sont pas des historiens des religions, hellénistes et autres spécialistes du monde antique déjà rompus aux méthodes et concepts que l'A. a développés depuis de nombreuses années pour étudier la littérature grecque, notamment dans les domaines de la linguistique discursive et de l'ethnopoétique.

Dans cette nouvelle version, l'ouvrage compte onze chapitres (dont trois nouveaux) encadrés par un avant-propos et une conclusion plus substantiels que dans l'édition originale, ainsi que des notes mises à jour qui se révèlent extrêmement riches et détaillées. Malheureusement, leur rejet en fin de volume ne facilite pas la lecture, tant leur consultation régulière s'avère indispensable pour suivre le raisonnement de l'A. Elles sont suivies d'une bibliographie, très complète et également mise à jour, s'étendant sur 61 pages $^{8}$, et d'un index général de 28 pages. Enfin, le volume se clôt sur une table des matières présentant des résumés synthétiques, fort utiles, de chacun des chapitres. Les extraits des différents textes étudiés au fil de l'ouvrage, qui étaient inclus en traduction française à la fin du volume de la Poétique des mythes dans la Grèce antique, ont été supprimés dans cette édition de poche: seules quelques citations en sont désormais inclues dans le corps du texte.

L'A. donne les raisons qui l'ont mené à publier cette nouvelle édition dans l'avantpropos. Le défaut des études contemporaines sur les mythes, souligne-t-il, réside dans leur tendance à n'envisager ces récits mythiques qu'à travers le schéma "canonique " de leur intrigue, sans réellement prendre en compte la diversité de leurs multiples versions. À cause de cette approche trop éloignée des sources, les dictionnaires et manuels de mythologie n'abordent que trop rarement les questions de «l'inépuisable 
richesse sémantique et figurée » et de la « remarquable efficacité sociale, religieuse et culturelle » de ces récits (p.7). Ce volume permet donc à l'A. de rappeler une nouvelle fois la double méthode linguistique et anthropologique qu'il estime être indispensable à l'étude des «mythes » (p. 13). Du côté de l'approche linguistique, il s'agit tout d'abord de prêter attention au "contexte d'énonciation du discours dans sa réalisation pragmatique» (linguistique discursive), ainsi qu'au «contexte culturel de représentations partagées, avec leur inévitable dimension symbolique " (analyse sémantique). Du côté de l'approche se revendiquant de l'anthropologie historique, il s'agit de comprendre l'impact pragmatique des «effets d'énonciation » et des «effets de sens » des récits étudiés. Enfin, l'A. insiste également sur la nécessité de l'approche ethnopoétique dont il a fait sa spécialité, qui permet de mieux comprendre ces récits héroïques ou «mythiques» en étudiant la performance rituelle «actée» (accompagnement chanté, joué ou dansé) qui accompagne très souvent leur narration'.

11 L'avant-propos donne également l'occasion à l'A. de revenir sur cet autre problème récurrent dans les études contemporaines sur la mythologie, soit la tendance à ontologiser la catégorie de mythe et à la considérer comme universelle, un point dont nous avons parlé au début de cet article. Par le passé, l'A. avait déjà eu plusieurs fois l'occasion de déplorer que des collègues hellénistes puissent encore être tentés de proposer une définition essentialiste du mythe ${ }^{10}$, et il s'inscrit une nouvelle fois en faux, dans le présent volume, contre deux tentatives récentes allant encore dans le même sens ${ }^{11}$. Dans l'édition de 2000 déjà, l'A. semblait considérer cette tendance comme l'illustration d'un manque de rigueur méthodologique dû à l'influence néfaste que le modèle économique néo-libéral exerce sur le monde académique depuis le début des années 1980. En dépréciant toute pensée et action politique centrée sur le lien social, ce modèle économique aurait affecté également l'étude des phénomènes sociaux et le développement des sciences humaines en général, entraînant l'érosion du "souci critique de la méthode partagée et de la cohérence épistémologique » essentielles à ces disciplines. L'A. attribue à cette détérioration le repli des chercheurs à l'intérieur de leur discipline, et leur manque de connaissance du contexte de l'anthropologie académique et du paradigme scientiste qui ont présidé à l'invention du concept de mythe. Quinze ans plus tard, la situation semble s'être aggravée, et ce pamphlet garde tout son caractère d'urgence (p. 17-19).

Il serait impossible de résumer dans le détail la démonstration que l'A. fait de sa méthode anthropologique et linguistique pour étudier les «mythes » à travers les onze chapitres de cet ouvrage. À vrai dire, les lecteurs habitués de ses recherches n'y trouveront rien de foncièrement nouveau. Dans les deux premiers chapitres, l'A. propose un argumentaire qu'il avait déjà développé dans de précédentes études ${ }^{12}$. Dans le premier, "Mythes et mytho-logiques ", il présente d'abord un historique succinct de la notion de "mythe " et de ce que le terme recouvre, depuis la Grèce antique (où ce que l'on qualifie aujourd'hui de mythes sont le plus souvent des récits fictionnels parlant d'un lointain passé, des archaîa et des palaiá) jusqu'à aujourd'hui. Puis dans "Créations narratives et poétiques", il rappelle l'importance d'étudier la forme poétique du mythe afin de mieux saisir quels effets concrets pouvait avoir sa récitation. L'exemple du récit du rapt de Perséphone est utilisé tout au long de ces deux chapitres, d'abord pour illustrer la variété des interprétations proposées par les différents courants d'étude, ensuite pour montrer la portée pragmatique de ce récit au sein de la culture grecque. Dans le reste de l'ouvrage, les six chapitres portant sur le traitement d'une figure héroïque ou divine dans différents textes (ou traditions iconographiques) 
de la tradition grecque sont restées quasiment les mêmes que dans la publication originale $^{13}$ : elles sont consacrées à Bellérophon, Clytemnestre et Oreste, Io et les Danaïdes, Hélène, Tirésias et Athéna, et enfin Artémis et Hippolyte. Chacune permet à l'A. d'illustrer un problème ou un effet particulier du récit étudié (articulations possibles entre un «mythe " étiologique et un rite, fonctionnement de la pragmatique interne à un récit, utilisation d'un « mythe » à des fins éducatives, etc.). À ces six études ont été ajoutés trois chapitres dans la même veine, reprenant les arguments d'articles déjà publiés par l'A. sur Thésée, Héraclès et Prométhée ${ }^{14}$. Le chapitre sur Thésée, notamment, permet à l'A. de compléter son approche en proposant une étude des sources iconographiques et non plus seulement textuelles de la tradition "mythique " liée à ce héros.

Pour couronner ces différentes études, l'A. revient dans sa conclusion sur la question du rôle joué par les "mythes" dans la Grèce antique : même si l'on peut considérer qu'ils étaient perçus avant tout comme des récits de fiction, créés de manière artisanale par les poètes et leurs successeurs logographes, la question de la croyance des Grecs en leurs mythes conserve sa pertinence. L'A. a recours à la notion de «feintise ludique (partagée) dans une fonction de satisfaction esthétique»(p.494), pour évoquer le plaisir que les Grecs pouvaient ressentir à choisir tous ensemble de "faire semblant " de croire à ces récits inventés, tout en soulignant que ce seul concept ne suffit pas à expliquer leur foisonnement et leur importance dans la société grecque antique. Les mythes grecs, rappelle-t-il, ne sont pas des récits fictifs mais des récits fictionnels, un terme soulignant le constant va-et-vient entre fictif et factuel de ces formes de discours, ni vrais ni faux. C'est cet aspect fictionnel, ajouté à leur dimension pragmatique, qui confère aux mythes grecs une certaine efficacité du point de vue éthique, politique, culturel ou religieux (p. 92-97 et p. 499-502).

La démonstration globale est convaincante, et l'A. espère dans sa conclusion (il le disait déjà en 2000) que le dialogue qu'il a proposé avec les récits grecs, mais aussi avec la théorie indigène sur ces récits, amènera à un autre dialogue critique avec les lecteurs modernes de ces récits, en suivant un itinéraire en opposition avec la tendance de l'éclectisme néo-libéral et les dérives naturalistes qu'il peut induire à propos du mythe. Quinze ans plus tard, l'A. renouvelle donc son appel avec cette édition de poche : mais participer à ce dialogue exige de maitriser parfaitement des outils de la linguistique discursive, de l'analyse sémantique, de l'anthropologie historique et de l'ethnopoétique. L'A. décrit son projet comme " animé par une volonté sans doute un peu pédante de méthode » (p. 502) : on craint en effet qu'il ne laisse le grand public sur le carreau.

\section{La Tortue et la lyre}

Ainsi qu'ils s'en expliquent dans l'introduction de leur ouvrage, l'enquête sur la «mythologie générative » que proposent John Scheid et Jesper Svenbro a été inspirée par une étude que Marcel Detienne avait consacrée à l'olivier en $1973^{15}$. Prenant pour point de départ un élément singulier, il avait démontré qu'un objet matériel pouvait à lui seul constituer une forme de noyau ou de matrice d'un "mythe». Scheid et Svenbro, suivant cette entreprise innovante, s'étaient demandé au début des années 1990 si l'existence d'un tel "mythe ", ainsi dépourvu de toute dimension narrative et condensé dans un objet, était seulement envisageable. Ils avaient entrepris de répondre 
à cette question avec la publication en 1994 du Métier de Zeus consacré à la mythologie du tissu ${ }^{16}$, mais également en s'associant pour donner, dans le cadre de l'École Pratique des Hautes Études de Paris, une série de séminaires communs intitulés «Comment le mythe et pourquoi ? ». Les enquêtes présentées dans ce nouvel ouvrage reprennent les dossiers qu'ils avaient réunis pendant la quinzaine d'années qu'ont duré ces séminaires, y compris un appendice au Collège de France en 2003.

16 Après une introduction dans laquelle les deux auteurs présentent la genèse de leur méthode et les fondements de leur réflexion théorique, l'ouvrage entraîne le lecteur, au fil de ses 229 pages, sur la piste de six types d'objets servant de matrice à la création de traditions mythiques, présentées dans autant de chapitres. L'ouvrage se conclut par un bref épilogue de 3 pages, suivi d'un appendice présentant en traduction française le texte de deux traditions relatives à Kérambos (Antoninus Liberalis, Métamorphoses, 22) et à Vertumne (Properce, Élégies IV, 2). Le volume comprend également une table des matières détaillée, mais aucun index ni bibliographie générale (les références auxquelles renvoient les auteurs sont indiquées directement dans les notes de bas de page).

17 Après avoir rendu hommage à Detienne et à son étude sur l'olivier, les deux auteurs passent en revue, dans l'introduction intitulée "Pour une mythologie générative », les autres influences qui les ont menés à mettre au point leur méthodologie. Il s'agit d'abord de l'étude de Louis Gernet sur les récits mythiques centrés autour d' " objets de valeurs » (agalmata $)^{17}$, dont Scheid et Svenbro se disent très proches dans leur tentative de se dégager de l' "hégémonie absolue du narratif dans le domaine de la mythologie » pour en venir à considérer également les objets ${ }^{18}$. C'est ensuite l'approche explicative de Jean-Pierre Vernant des mythes grecs à travers les noms propres de leurs protagonistes $^{19}$, à la suite de Lévi-Strauss; par exemple đEdipe (ô̂dos « [pied] enflé » mais aussi oîda « je sais ») dont le nom " contiendrait la tragédie condensée comme une espèce de bouillon en cube » et aurait précisément orienté la construction du récit par Sophocle et ses précurseurs (p. 20).

Pour les deux auteurs, influencés par Gernet autant que par Vernant, c'est avant tout par son nom que l'objet devient "générateur de mythes ", comme dans le cas du récit de la ruse de Didon/Élissa fondant la première citadelle de Carthage, Bursa, à partir d'une peau de vache (bursa en grec) ${ }^{20}$. Contrairement à ce que raconte le récit grec, ce n'est pas la cité de Carthage qui a été baptisée Bursa en mémoire de la peau de vache utilisée pour sa fondation, mais bien le toponyme (non grec?) Borsa qui a suggéré à l'oreille grecque le mot bursa et a servi de base à la composition du mythe. La démarche générative de l'objet est ici soutenue par la charge symbolique qui rattache, pour un Grec, la " peau de vache » à la " cité » (toute cité grecque est fondée par le sacrifice d'un bovidé). Ce que nous appelons un «mythe » serait en fin de compte « ces associations symboliques préexistantes qui servent de matrice à l'élaboration des récits mythologiques plutôt que les récits eux-mêmes » (p. 17). Comme dans Le Métier de Zeus, Scheid et Svenbro proposent donc de comprendre le mythe non comme un récit, une image ou un rituel, mais comme une "concaténation de catégories ", un "agrégat de significations ", la matrice ou le noyau porteur du récit, de l'image ou du rituel mythique (p. 22). Finalement, la position revendiquée par Scheid et Svenbro consiste à dire que "les mythes se fabriquent avec des mots, non pas avec des idées", en établissant un parallèle avec la méthode de "génération " poétique à partir des mots prônée par Stéphane Mallarmé et Paul Valéry (p. 30-31). 

de cités » à propos de Carthage, déjà mentionnés dans l'introduction, et d'Alexandrie, où la farine utilisée en lieu et place de la craie pour tracer les frontières de la cité en devenir sert de support symbolique pour raconter sa fertilité future. La deuxième, "Tissus mythiques ", convie à un bref parcours à propos de la dimension symbolique prêtée à différentes pièces de tissus allant du peplos d'Héra au manteau de Syloson et à l'ex-voto d'Horace. Le troisième chapitre propose une courte réflexion sur les «Études de botanique: Jacinthe, Crocus et Sidè ", sur l'importance d'avoir connaissance des savoirs botaniques grecs pour comprendre les mythes "floraux", racontant par exemple l'origine de la hyacinthe et du crocus safrané, des fleurs nées du sang des jeunes gens dont elles portent le nom, tous deux tués après avoir été accidentellement frappés à la tête par un disque en pierre mal lancé. Ou encore les rapports symboliques de la grenade - sidè, nom de l'épouse d'Orion - avec les liens du mariage. Le quatrième chapitre, plus long, est celui qui donne son titre au volume (« De la pierre à la lyre. La tortue dans tous ses états ») : il se concentre sur la symbolique de la tortue, de la pierre (tombale) et de la lyre, et sur les rapports entre la lyre/tortue et le monde des morts dans plusieurs récits (Orphée et la mort d'Eschyle). Le cinquième, «Destinées de musiciens", mêle les réflexions des deux précédents chapitres en abordant les différents mythes jouant entre connaissances zoologiques antiques et noms de ceux qui jouent de la lyre, en l'occurrence le berger Kérambos (transformé en lucane cerf-volant auquel il donne son nom, insecte dont la tête ressemble à une lyre) et Orphée «le mérou " (orphos, poisson censé continuer à vivre même après avoir été découpé en morceaux, comme la tête d'Orphée). Enfin, le sixième chapitre, "La force du nom ", revient sur le nom propre en tant que générateur de mythes à l'aide de trois exemples. D'abord le nom d'Ajax, que l'étymologie propose de rattacher soit à l'expression de lamentation que le héros achéen, dans la tragédie de Sophocle, met lui-même en relation avec son nom au moment de sa mort (AIAI), soit au nom de l'aigle apparu à sa naissance (aietos), soit à la " honte » (aidôs) qui le pousse au suicide, parmi beaucoup d'interprétations antiques et modernes. Avec Héraclès, les auteurs explorent du côté grec le paradoxe du nom de « kleos d'Héra " porté par un héros que la déesse déteste en suivant la piste de l'amphisémie du terme kleos, qui peut désigner aussi bien la gloire que la honte; du côté romain, ils relèvent la manière dont des traditions relatives au passage d'Hercule au Forum Boarium sont construites en écho à ses épiclèses et surnoms grecs. Enfin, avec le dieu Vertumne chanté par Properce et Ovide, la dernière enquête s'ancre dans le monde romain.

Dans un très court mais stimulant épilogue (p. 215-217), les deux auteurs formulent plusieurs remarques qu'il vaut la peine de mentionner ici. Ils soulignent notamment l'importance, pour eux, de parler de « poétique » plutôt que de « théorie » du mythe, un terme qui pourrait laisser penser qu'ils s'intéressent à la «nature du fait mythique en général ». En réalité, leur étude concerne la construction, l'élaboration et la mise en place de récits, rituels, images et exégèses mythiques, sans établir entre eux une hiérarchie qui ferait de l'un (par exemple le récit) l'explication de l'autre (par exemple le rituel). Autre remarque d'importance formulée par les A. à la suite de leurs enquêtes : ils remarquent que, du côté grec comme du côté romain, les mythes sur lesquels ils ont travaillé sont inscrits dans un imaginaire plutôt archaïque, alors qu'ils sont en réalité connus par des documents de date plutôt récente, hellénistique, sans qu'il soit pour autant possible de rejeter pour ces récits l'appellation de mythes pour les qualifier de "légendes" ou de "constructions tardives». Scheid et Svenbro en 
concluent qu'il n'existe pas d'époque spécifiquement "mythopoiétique», pas de période où la "pensée mythique » aurait été plus fertile : autrement dit, il n'y a pas eu, en Grèce, de période d'invention du mythe par opposition à une période d'exégèse. Voilà une constatation particulièrement bienvenue alors que tout un pan de la recherche académique dans le domaine des mythes cherche encore et toujours à reconstituer ou retrouver les « versions originales» des mythes, celles qui auraient été composées illo tempore et seraient porteuses du message mythique dans toute sa vérité.

\section{Mythes (re)configurés}

21 Le parallèle entre composition poétique et composition mythique proposé par Scheid et Svenbro ("génération » des récits à partir des mots plutôt qu'à partir des idées) nous permet d'établir un lien avec le dernier ouvrage recensé dans le présent article, qui se distingue en effet des trois autres volumes que nous avons examinés en ceci qu'il associe à la réflexion académique la voix d'une artiste contemporaine pour discuter du processus de ce qu'elle appelle le «surgissement/détournement des mythes». Cet ouvrage collectif est le fruit de différents dialogues qui ont eu lieu au Centre de recherche en langues et littératures européennes comparées (CLE) de l'Université de Lausanne, entre 2000 et 2010. Ce volume publié sous la direction de trois enseignantes du CLE, Ute Heidmann (professeure de littérature comparée), Maria Vamvouri Ruffy (helléniste et chargée de cours) et Nadège Coutaz (doctorante et assistante auprès du CLE et des Facultés Saint-Louis de Bruxelles) s'inscrit dans la droite lignée de l'étude des mythes prônée par Claude Calame. Et pour cause, Ute Heidmann raconte comment, lors d'un séminaire mené conjointement avec Claude Calame sur les Poétiques comparées des mythes en 2000, l'idée lui est venue d'inviter aux discussions la poète et dramaturge Sylvie Dupuis, discussions qui se sont par la suite enrichies avec la participation de nombreux autres intervenants et l'organisation de plusieurs colloques et journées de discussions.

Le volume de 255 pages, précédé d'un sommaire et d'une introduction de 9 pages rédigée à trois voix par ses co-éditrices, est organisé en trois parties. La première, «Parcours exploratoires et dialogiques» commence avec deux essais de Sylvie Dupuis. Dans "Surgissement/détournement des mythes dans la pratique poétique ", l'artiste raconte comment elle utilise et se réapproprie des mythes dans le cadre de son processus d'écriture poétique. Dans son deuxième essai, «Sources et genèse des Enfers ventriloques ", l'artiste dévoile une nouvelle fois les coulisses du processus de création, cette fois pour l'écriture d'une pièce de théâtre. Ces deux incursions dans le monde intérieur d'un artiste sont suivies de quatre études plus académiques réagissant chacune de l'extérieur à une œuvre théâtrale, poétique et cinématographique contemporaine. Il s'agit de "Échos polyphoniques aux Enfers ventriloques de Sylviane Dupuis » par Sophie Klimis ; "Comparaison "différentielle" et scène(s) de parole. Le double recours à Eurydice dans l'œuvre poétique de Sylviane Dupuis" par Ute Heidmann ; "Sortir de l'ombre du mythe: Oreste chez Eschyle, Sartre et Ritsos » par Mari Vamvouri Ruffy ; et « Perséphone ravie aux enfers. (R)écrire (sic!) les mythes sous l'oppression : Yannis Ritsos et Sándor Weöres » par Myriam Olah.

23 La deuxième partie, "Parcours du continent Antigone", est consacrée aux liens entretenus entre la tragédie de Sophocle et ses différentes réinterprétations modernes, dans des études menées du point de vue de la philologie, de la littérature comparée et 
de la théorie du cinéma : "En lugar del varón. Antígona en Sófocles y en Zambrano » par Luis Miguel Pino Campos; "L'impact du genre. La tumba de Antígona de María Zambrano, (r)écriture (sic!) de Sophocle » par Nadège Coutaz; et « Un film de guerre. Antigone de Danièle Huillet et Jean-Marie Straub » par Benoît Turquety.

Enfin, la troisième partie "Parcours (inter-)disciplinaires" nous ramène dans le domaine plus familier des études classiques avec deux contributions sur l'étude des mythes antiques : d'abord des récits grecs avec « Pour une anthropologie historique des récits héroïques grecs: comparaison différentielle et pragmatique poétique» par Claude Calame, dans laquelle on reconnaît quelques-uns des arguments développés également dans Qu'est-ce que la mythologie grecque?, même si l'article propose ici une analyse critique plus détaillée de la lecture structurale des mythes et des défauts d'une narratologie comparative qui omettrait de considérer les aspects pragmatiques des récits étudiés. Le deuxième article, «Sur la réception à Rome de deux images-clefs du prologue de la Médée d'Euripide : les roches Symplégades et les pins du Pélion » par Jacqueline Fabre-Serris, traite de la réécriture des mythes grecs à l'époque romaine et fait la démonstration des liens intertextuels entre différentes versions grecques et latines. Le dernier essai du volume, "Études des imaginaires mythiques et interdisciplinarité » par Myriam Watthée-Delmotte - fondatrice du Centre de Recherche sur l'Imaginaire de l'Université catholique de Louvain-la-Neuve - propose une réflexion sur l'importance de prendre en compte le contexte, le médium poétique et l'ancrage ritualisé dans l'étude des recréations et réinterprétations de mythes dans la littérature occidentale afin de mieux comprendre le rôle identitaire qu'elle joue dans la reconnaissance d'une communauté culturelle, même si les références aux sources antiques ne sont parfois plus reconnues par le lectorat. Le volume se clôt sur un index des noms propres (6 pages) et les notices bio-bibliographiques des contributeurs (4 pages).

25 Cet ouvrage souffre en partie des défauts partagés par beaucoup de volumes collectifs en ce qu'il lui manque une véritable introduction méthodologique et surtout une forme de conclusion. La plupart des contributions sont très clairement adressées à un public académique, mais permettent d'avoir un bon aperçu des travaux et des discussions qui ont animé pendant une dizaine d'années ce Centre de recherche en langues et littératures européennes comparées, et de ce que peut produire une collaboration entre philologues classiques et chercheurs en littérature comparée. Mais son grand succès réside surtout dans l'idée d'avoir invité un artiste à s'exprimer sur le processus de création poétique, dans un exercice qui se révèle être une fenêtre ouverte sur le fonctionnement de la pragmatique du mythe. La possibilité d'entrer ainsi au cœur d'une création poétique contemporaine permet notamment de démontrer de façon fort intéressante le bienfondé de la constatation de Scheid et Svenbro citée plus haut à propos de l'impossibilité de définir, dans l'Antiquité, une " période mythopoiétique » qui aurait vu la création de tous les mythes, par opposition à des périodes d'interprétation et d'exégèse. Ainsi que Sylvie Dupuis le souligne au début de son premier essai, le mythe n'existe, depuis toujours, «qu'à travers ses métamorphoses, ses réinterprétations et ses détournements successifs » (p. 1). Ainsi que le dit l'éditrice principale de ce volume, les mythes ne sont jamais porteurs d'un sens inhérent, mais ce dernier est généré en perpétuelle réinvention à partir de la situation du sujet énonciateur (p. II).

26 La lecture de ces quatre ouvrage met en lumière que la tendance à étudier les récits héroïques qualifiés de mythes en les réduisant à de vagues schémas conduit à oublier la 
diversité de leurs sources et de leurs variantes, mais aussi à perdre de vue leur contexte de production artistique/artisanale (poétique/poiétique), leur pragmatique et leur efficacité symbolique. Mais les fondements de cette méthode - notamment l'affirmation que la catégorie de "mythe " est une invention récente et qu'elle ne saurait renvoyer à une classification universelle - est loin d'être acceptée par tous les chercheurs au sein des différentes disciplines ${ }^{21}$, et les objectifs qu'elle poursuit ne suscitent la plupart du temps pas un grand intérêt de la part du grand public, ni même du reste du monde académique. À ce sujet, il semble que l'ouvrage introductif de Pauline Schmitt Pantel, présentant cette méthode sans avoir recours au jargon très spécialisé de Claude Calame, est déjà un pas en direction de sa potentielle diffusion.

En réalité, c'est surtout la présence de "motifs » narratifs similaires dans différents récits qui ne cesse de fasciner à propos des mythes : ces motifs sont-ils le résultat d'une transmission transculturelle (diffusionnisme), ou bien le produit d'un mode de fonctionnement de l'esprit humain qui serait similaire dans toutes les cultures (théorie des archétypes)? Ce sont là des questions que prétendent actuellement résoudre certains sanskritistes, préhistoriens et chercheurs en études folkloriques, qui proposent une méthode mêlant structuralisme lévi-straussien, mythologie comparée de Dumézil, linguistique indo-européenne et génétique des populations pour étudier les origines et la diffusion des $«$ mythes $»^{22}$.

De telles approches, en dépit des nombreux problèmes méthodologiques qu'elles soulèvent ${ }^{23}$, rencontrent un grand succès notamment dans la presse généraliste ${ }^{24}$. Elles vont bien sûr à l'encontre de toutes les précautions recommandées par les spécialistes dont on a présenté les ouvrages. C'est que, comme le rappelle Calame, réduire des récits "mythiques » aux éléments essentiels de leur intrigue, en effaçant leurs spécificités sémantiques, n'est en réalité qu'un subterfuge académique ${ }^{25}$. Cela permet certes de comparer entre eux les « motifs » que l'on a ainsi créés artificiellement, mais dans des conditions d'abstraction et de généralisation telles que cette manière de les étudier ne peut produire aucune conclusion valable et n'a guère qu'une valeur opératoire. Au-delà du schéma narratif commun, il faut également tenir compte des différences dans la tournure des événements racontés, des différents protagonistes et de leur situation ou encore des intentions de l'A. Calame admet toutefois qu'à l'issue de sa démonstration le chercheur se trouve confronté à une nouvelle série de questions: entre l'action pratique (rite) de l'homme ou l'action narrative et fictionnelle (mythe), laquelle est la première et inspire l'autre ? Les deux seraient-elles orientées par un dispositif neuronal et cognitif relatif à la structure du cerveau humain ? Ce dispositif serait-il universel, et susceptible d'être étudié par une approche inspirée par les sciences cognitives ${ }^{26}$ Sur le même sujet, Scheid et Svenbro relèvent qu'il n'est pas toujours possible d'expliquer la présence d'un « thème » narratif identique dans plusieurs traditions comme le résultat d'une diffusion. Mais ils soulignent eux aussi que l'exercice auquel doit avant tout se livrer un historien des religions est celui de l'étude de chaque thème dans le contexte culturel qui lui est propre. Ce n'est qu'à cette condition qu'il serait possible d'envisager dans un second temps la comparaison entre les mêmes thèmes dans différentes cultures, sous un angle résolument contrastif ${ }^{27}$.

Mais ni Calame, ni Scheid et Svenbro ne vont plus loin dans leurs réflexions à ce sujet et le projet d'une comparaison différentielle ou contrastive de ces "motifs » littéraires reste au stade d'idée potentielle. Nul ne semble vouloir prendre le risque de se lancer dans l'étude des variantes entre plusieurs versions du mythe au sein de différentes 
cultures. L'entreprise serait-elle trop risquée ? Après l'échec du comparatisme indoeuropéen de Dumézil, est-il possible, voire même souhaitable de se lancer comme l'a fait Michael Witzel dans de telles comparaisons à l'échelle transculturelle, voire mondiale? Quoi qu'il en soit, les quatre ouvrages recensés dans le présent article le démontrent bien: l'étude des "mythes", de leurs créations et de leur réception produit d'abord des résultats quand on prête une attention minutieuse à leur contexte de production. Si elle est possible, une comparaison «contrastive» dans ce domaine doit être nécessairement limitée et proche des sources, mais également proposer des réponses plus nuancées au problème posé par la prétendue universalité de certains schémas qui se révèlent souvent moins mythiques que construits comme tels par les savants $^{28}$.

\section{NOTES}

1. .R. GRAVES, The Greek Myths, Harmondsworth, Penguin Books, 1955 (trad. française Les Mythes grecs, Paris, Fayard, 1967 et nombreuses rééditions).

2. .E. HAmiLton, Mythology. Timeless Tales of Gods and Heroes, Little, Brown and Company, 1942 (trad. française La Mythologie. Ses dieux, ses héros, ses légendes, Paris, Marabout, 1978 et nombreuses rééditions successives). L'ouvrage est régulièrement présenté dans ses multiples éditions comme « le plus clair et le plus complet sur la mythologie».

3. .J. CAMPBELL, The Hero with a Thousand Faces, New York, Pantheon Books, 1949 (trad. française Le Héros aux mille et un visages, Paris, Robert Laffont, 1977 ; traduction française la plus récente chez J'ai lu, coll. «Bien-être », 2013). Selon la théorie formulée par Joseph Campbell, l'ensemble des mythes de l'humanité raconterait en réalité une seule histoire, un "monomythe » narrant le «voyage du héros» (hero's journey) dont la structure séparée en plusieurs étapes (séparation, initiation, retour du héros) serait perceptible derrière chaque variante de chaque récit dans le monde. La théorie est largement réfutée dans le monde académique (voir R.A.SEGAL, Joseph Campbell: An Introduction, New York, Garland Publishing, 1987), mais a rencontré un succès considérable auprès du grand public aux États-Unis et, plus récemment, dans le monde francophone. Le réalisateur George Lucas, notamment, avait déclaré avoir suivi ce schéma décrit par Campbell pour écrire l'intrigue de la trilogie originale de Star Wars ; cf. J.S. LAWRENCE, « Joseph Campbell, George Lucas, and the Monomyth ", in M. KAPELL, J.S. LAWRENCE (éd.), Finding the Force of the Star Wars Franchise: Fans, Merchandise, \& Critics, New York, Lang, 2006, p. 2134.

4. .A. VAN GENNEP, La Formation des légendes, Paris, Flammarion, 1929 ; V. PROPP, Morphologie du conte ; suivi de Les Transformations des contes merveilleux, Paris, Seuil, 1970 ; V. PROPP, Les Racines historiques du conte merveilleux, Paris, Gallimard, 1983. Eux-mêmes doivent une bonne partie de leur inspiration aux travaux des frères Grimm; voir entre autres N. BELMONT, Paroles païennes : mythe et folklore: des frères Grimm à P. Saintyves, Paris, Imago, 1986; J.zIPES, The Brothers Grimm: From Enchanted Forests to the Modern World, Second Edition, Palgrave Macmillan, 2002. Les définitions des catégories de " mythe ", " conte » et « légende » varient selon les auteurs et les courants. Le plus souvent (chez les frères Grimm notamment), néanmoins, on comprend le mythe comme un récit fantastique dont l'origine se perdrait dans la nuit des temps; le conte en serait un vestige dépouillé de son caractère sacré et de sa fonction religieuse, transmis par la tradition orale; la 
légende, enfin, serait un récit fantastique plus récent et local, ancré dans un événement et des figures historiques.

5. .R. BARTHES, Mythologies, Paris, Seuil, 1957. De Lévi-Strauss, on retient en général sa phrase "Quelle que soit notre ignorance de la langue et de la culture de la population où on l'a recueilli, un mythe est perçu comme mythe par tout lecteur dans le monde entier. La substance du mythe ne se trouve ni dans le style, ni dans le mode de narration, ni dans la syntaxe, mais dans l'histoire qui y est racontée " («La structure des mythes», Anthropologie structurale, Paris, Plon, 1974 [1958], p. 232). Ainsi que le font remarquer John Scheid et Jesper Svenbro dans l'ouvrage recensé infra, « on peut se demander si la position défendue par Lévi-Strauss n'est pas simplement due au fait qu'il a été contraint de travailler sur des mythes qui provenaient d'un nombre considérable de langues différentes, impossibles à maîtriser pour un seul chercheur, bref sur des mythes qu'il n'a pu connaître qu'à travers des traductions.» (La tortue et la lyre, p. 29). Mais à propos de la définition du mythe, rappelons toutefois que Lévi-Strauss émet également des doutes dans ses travaux ultérieurs sur la pertinence du concept: « Nous rejetons, en effet, les opinions trop hâtives sur ce qui est mythique et ce qui ne l'est pas, et revendiquons pour notre usage toute manifestation de l'activité mentale ou sociale des populations étudiées (...)» (Le Cru et le cuit [Mythologiques*], Paris, Plon, 1964, p. 12). Voir G. SALMON, Les Structures de l'esprit : Lévi-Strauss et les mythes, Paris, PUF, 2013.

6. .Les études fondatrices sont notamment celles de M. DETIENNE, L'invention de la mythologie, Paris, Gallimard, 1981 ; P. VEYNE, Les Grecs ont-ils cru à leurs mythes? Essai sur l'imagination constituante, Paris, Seuil, 1983 ; c. CALAME, Mythe et histoire dans l'Antiquité grecque. La création symbolique d'une colonie, Paris, Les Belles Lettres, 2011 (1 ${ }^{\text {ère }}$ édition : Lausanne, Payot, 1996) ; B. LINCoLN, Theorizing Myth. Narrative, Ideology, and Scholarship, Chicago/London, University of Chicago Press, 1999.

7. .c. CALAME, Poétique des mythes dans la Grèce antique, Paris, Hachette, 2000, p. 7.

8. .On regrettera que les ouvrages d'un même auteur ne soient pas classés par ordre chronologique; dans ces conditions il est difficile, par exemple, de retrouver la référence d'un article précis de Claude Calame parmi la quarantaine de ses études recensées dans la bibliographie.

9. .Sur l'ethnopoétique, l'auteur renvoie à la dernière publication qu'il a dirigée sur ce sujet avec F. DUPONT, B. LORTAT-JACOB, M. MANCA, La Voix actée. Pour une nouvelle ethnopoétique, Paris, Kimé, 2010.

10. .Dans son « Avant-propos II (2011) » de la deuxième édition de Mythe et histoire dans l'Antiquité grecque, 2011, o.c. (n. \$), p. 15, l'auteur citait les exemples d'E. CSAPO, Theories of Mythology, 2005, p. 62 et de S.DES BOUVRIES, "The Definition of Myth. Symbolic Phenomena in Ancient Greek Culture ", in S. DES Bouvries (éd.), Myth and Symbol I. Symbolic Phenomena in Ancient Greek Culture, Bergen, Norwegian Institute at Athens, 2002, p. 62.

11. .G. CASADIO, "Mythos vs. mito. Mythos vs myth», Minerva 22 (2009), p. 41-63; R. L. FOWLER, «Mythos and logos », JHS 131 (2011), p. 45-66. Voir aussi le compte rendu rédigé par Cl. Calame cidessous, p. 403-414.

12. On y retrouve notamment la matière présentée dans le premier chapitre de la Poétique des mythes publié en 2000 et dans le chapitre «Illusions de la mythologie » de Mythe et histoire dans l'Antiquité grecque, 2011, o.c. (n. \$), p. 19-89.

13. Voir à leur sujet les différents comptes rendus de la Poétique des mythes, par exemple R. BUXTON, JHS 121 (2001), p. 178-179 ; R. LE MER, ASSR 120 (2002), p. 63-126 ; M. MUND-DOPCHIE, AC 72 (2003), p. 304-305 ; P.N. HERNÁNDEZ, « Calame and Detienne on Myth », CR 55 (2005), p. 500-502.

14. .Les trois nouveaux chapitres (numérotés VII, VIII et IX dans ce livre) sont adaptés des articles suivants: "Thésée l'Athénien au Metropolitan Museum of Art de New York: scènes étiologiques de légitimation et questions de méthode ", in D. YARTOMANOLAKIS (dir.), An Archaeology of Representations. Ancient Greek Vase-Painting and Contemporary Methodologies, Athènes, Kardemitsa, 2009, p. 98-127; « Héraclès, animal et victime sacrificielle dans les Trachiniennes de Sophocle », in 
C. BONNET et al. (éd.), Le Bestiaire d'Héraclès, Liège, CIERGA, 1998 (Kernos, suppl. 7), p. 197-215 ; « The Pragmatics of "Myth" in Plato's Dialogues: the Story of Prometheus in the Protagoras", in C. COLlOBERT et al. (éd.), Plato and Myth. Studies in the Use and Status of Platonic Myths, Leiden/Boston, Brill, 2012, p. 127-143.

15. .M. DETIENNE, « L'olivier : un mythe politico-religieux », in M. FINLEY (éd.), Problèmes de la terre en Grèce ancienne, Paris/La Haye, 1973, p. 293-306.

16. J. SCHEID, J. SVENBRO, Le Métier de Zeus: mythe du tissage et du tissu dans le monde gréco-romain, Paris, La Découverte, 1994 [Paris, Errance, 2003].

17. .L. GERNET, « La notion mythique de valeur en Grèce ancienne », Journal de Psychologie 41 (1948), p. 415-462 [repris dans Anthropologie de la Grèce antique, Paris, Maspero, 1968, p. 293-306.

18. .sCHEID - SVENBRO, Le Métier de Zeus, p. 10.

19. J.-P. VERNANT, P. VIDAL-NAQUET, Mythe et tragédie en Grèce ancienne, Paris, Maspero, 1972, p. 113.

20. .Pour une réflexion parallèle sur les liens entre toponymie et mythogenèse, voir P. SAUZEAU, "Toponymie, idéologie et mythologie », in A. GASSE et al. (éd.), Et in Aegypto et in Aegyptum. Recueil d'études dédiées à Jean-Claude Grenier, vol. 4, Montpellier, Université Paul-Valéry III/CNRS, 2012 (Cahiers de l'ENiM, 5), p. 691-698.

21. .La polémique, par bien des aspects, fait écho à celle entre les approches essentialistes et constructivistes de la catégorie « religion ».

22. .Notamment M. WITZEL, The Origins of the World's Mythologies, Oxford, OUP, 2012, ou les différents articles proposés dans la revue franco-belge Nouvelle Mythologie Comparée / New Comparative Mythology [http://nouvellemythologiecomparee.hautefort.com].

23. À propos des problèmes posés par la méthode de M. Witzel, par exemple, on renverra au compte rendu de The Origins of the World's Mythologies publié par Bruce Lincoln dans Asian Ethnology 74 (2015), p. 443-449. Lincoln développe plus en détail cette critique dans la conférence « History, Comparison, and the History of Comparatism in light of a recent effort », présentée en septembre 2015 dans le cadre du Sawyer Seminar à l'Université de Cambridge (parution à venir en tant que chapitre d'un futur ouvrage de Bruce Lincoln sur le comparatisme, pour l'instant intitulé Apples and Oranges: Toward a Weaker Comparative Method ).

24. .Ainsi, dans le monde francophone, deux numéros spéciaux de revues publiés à peu près en même temps que les ouvrages recensés dans cet article adoptent une approche présentant le mythe comme une forme de récit transcendant, indépendant de tout support textuel, dont certains seraient communs à toute l'humanité. Il s'agit du hors-série 21 du Monde des religions (décembre 2013) intitulé Les Grands mythes de l'humanité (avec une introduction prudente de Walter Burkert), et du Cahier de Science \& Vie numéro 147 (juillet 2014) sur L'Origine des mythes. Pourquoi l'humanité partage les mêmes histoires (où figurent parmi les spécialistes invités certains des «mythologues » de la revue Nouvelle Mythologie Comparée).

25. .CALAME, dans Qu'est-ce que la mythologie grecque?, en particulier p.131-137 (chapitre déjà présent dans la Poétique des mythes en 2000), développe cet élément de réflexion à partir du récit homérique de Bellérophon rejetant les avances d'Antéia, la femme du roi Proïtos, dont on peut comparer le motif avec celui du récit biblique de Joseph et de la femme de Potiphar ou avec celui d'Hippolyte et Phèdre dans les deux tragédies d'Euripide, entre autres parallèles.

26. .CALAME, Qu'est-ce que la mythologie grecque?, p. 501-502.

27. .sCHEID - SVENBRo, La Tortue et la lyre, p. 38-39. Les auteurs parlent ici du «thème de Didon », la ruse que la reine phénicienne met en œuvre pour fonder Byrsa (la future Carthage). Alors qu'elle avait demandé aux rois indigènes de la Numidie où elle était arrivée qu'on lui fasse don de la terre qu'elle pourrait recouvrir (tenere) d'une peau de vache (bursa), la reine découpe ensuite en très fines lanières cette peau et s'en sert pour entourer (autre sens de tenere) un immense territoire. Le thème se retrouve aussi bien chez les Hottentots que les Kirghiz, en Scandinavie et en Asie du Sud-Est. 
28. .Sur la méthode comparative, voir C. CALAME, B. LINCOLN (éd.), Comparer en histoire des religions antiques: controverses et propositions, Liège, Presses Universitaires de Liège, 2012 et le futur ouvrage de Bruce Lincoln mentionné supra (Apples and Oranges. Toward a Weaker Comparative Method). Il sera également intéressant, quand elles seront publiées, de prendre connaissance des différentes études menées dans le cadre du projet Wandering Myths à l'Université d'Oxford (L. AUDLEY-MilleR, B. DIGNAS (éd.), Wandering Myths. Transcultural Uses of Myth in the Ancient World, Berlin, De Gruyter, parution prévue en février 2017)

\section{AUTEURS}

\section{PHILIPPE MATTHEY}

Fonds national de la recherche scientifique suisse - Université de Genève - Université de Liège 